\title{
THE BANACH ALGEBRA OF CONTINUOUS BOUNDED FUNCTIONS WITH SEPARABLE SUPPORT
}

\author{
M. R. KOUSHESH
}

\begin{abstract}
We prove a commutative Gelfand-Naimark type theorem, by showing that the set $C_{S}(X)$ of continuous bounded (real or complex valued) functions with separable support on a locally separable metrizable space $X$ (provided with the supremum norm) is a Banach algebra, isometrically isomorphic to $C_{0}(Y)$, for some unique (up to homeomorphism) locally compact Hausdorff space $Y$. The space $Y$, which we explicitly construct as a subspace of the Stone-Čech compactification of $X$, is countably compact, and if $X$ is nonseparable, is moreover non-normal; in addition $C_{0}(Y)=C_{00}(Y)$. When the underlying field of scalars is the complex numbers, the space $Y$ coincides with the spectrum of the $\mathrm{C}^{*}$-algebra $C_{S}(X)$. Further, we find the dimension of the algebra $C_{s}(X)$.
\end{abstract}

\section{INTRODUCTION}

Throughout this article the underlying field of scalars (which is fixed throughout each discussion) is assumed to be either the real field $\mathbb{R}$ or the complex field $\mathbb{C}$, unless specifically stated otherwise. All spaces considered are assumed to be Hausdorff. Let $X$ be a completely regular space. Denote by $C_{b}(X)$ the set of all continuous bounded functions on $X$. If $f \in C_{b}(X)$, the zero-set of $f$, denoted by $\mathrm{Z}(f)$, is $f^{-1}(0)$, the cozero-set of $f$, denoted by $\operatorname{Coz}(f)$, is $X \backslash \mathrm{Z}(f)$, and the support of $f$, denoted by $\operatorname{supp}(f)$, is $\mathrm{cl}_{X} \operatorname{Coz}(f)$. Let

$$
\operatorname{Coz}(X)=\left\{\operatorname{Coz}(f): f \in C_{b}(X)\right\} .
$$

The elements of $\operatorname{Coz}(X)$ are called cozero-sets of $X$. Denote by $C_{0}(X)$ the set of all $f \in C_{b}(X)$ which vanish at infinity (i.e., $|f|^{-1}([\epsilon, \infty))$ is compact for each $\epsilon>0$ ) and denote by $C_{00}(X)$ the set of all $f \in C_{b}(X)$ with compact support.

The purpose of this article is to show that the set $C_{s}(X)$ of continuous bounded (real or complex valued) functions with separable support on a locally separable metrizable space $X$ (provided with the supremum norm) is a Banach algebra, which is isometrically isomorphic to the Banach algebra $C_{0}(Y)$, for some unique (up to homeomorphism) locally compact space $Y$. (This is a direct conclusion of the commutative Gelfand-Naimark Theorem when the underlying field of scalars is $\mathbb{C}$, provided that one assumes $C_{s}(X)$ to be a Banach algebra.) The space $Y$, which is explicitly constructed as a subspace of the Stone-Cech compactification of $X$, is shown to be countably compact and non-normal (for the latter, provided that $X$

2010 Mathematics Subject Classification. Primary 46J10, 46J25, 46E25, 46E15; Secondary 54C35, 54D35, 46H05, 16S60.

Key words and phrases. Gelfand theory; Stone-Čech compactification; Real Banach algebra; Separable support; Locally separable metrizable space; Spectrum; Functions vanishing at infinity; Functions with compact support.

This research was in part supported by a grant from IPM (No. 90030052). 
is non-separable) and moreover $C_{0}(Y)=C_{00}(Y)$. In the case when the underlying field of scalars is $\mathbb{C}$, the space $Y$ coincides with the spectrum of the $\mathrm{C}^{*}$-algebra $C_{s}(X)$. Further, the dimension of the algebra $C_{s}(X)$ is found in terms of the density of $X$.

We now review briefly some known facts from the theories of the Stone-Čech compactification and metrizable spaces. Additional information on these subjects may be found in $[3$ and $[4$.

1.1. The Stone-Čech compactification. Let $X$ be a completely regular space. The Stone-Čech compactification $\beta X$ of $X$ is the compactification of $X$ which is characterized among all compactifications of $X$ by the following property: Every continuous $f: X \rightarrow K$, where $K$ is a compact space, is continuously extendable over $\beta X$; denote by $f_{\beta}$ this continuous extension of $f$. Use will be made in what follows of the following properties of $\beta X$. (See Sections 3.5 and 3.6 of 3 .)

- $X$ is locally compact if and only if $X$ is open in $\beta X$.

- Any open-closed subspace of $X$ has open-closed closure in $\beta X$.

- If $X \subseteq T \subseteq \beta X$ then $\beta T=\beta X$.

- If $X$ is normal then $\beta T=\operatorname{cl}_{\beta X} T$ for any closed subspace $T$ of $X$.

1.2. Separability and local separability in metrizable spaces. The density of a space $X$, denoted by $d(X)$, is the smallest cardinal number of the form $|D|$, where $D$ is dense in $X$. Therefore, a space $X$ is separable if $d(X) \leq \aleph_{0}$. Note that in any metrizable space the three notions of separability, being Lindelöf, and second countability coincide; thus any subspace of a separable metrizable space is separable. A space is called locally separable if each of its points has a separable open neighborhood. By a theorem of Alexandroff, any locally separable metrizable space $X$ can be represented as a disjoint union

$$
X=\bigcup_{i \in I} X_{i},
$$

where $I$ is an index set, and $X_{i}$ is a non-empty separable open-closed subspace of $X$ for each $i \in I$. (See Problem 4.4.F of [3].) Note that $d(X)=|I|$, provided that $I$ is an infinite set.

\section{The Banach algebra $C_{s}(X)$ of Continuous bounded functions with SEPARABlE SUPPORT ON A LOCALly SEPARABlE METRIZABLE SPACE $X$}

Definition 2.1. For any metrizable space $X$ let

$$
C_{s}(X)=\left\{f \in C_{b}(X): \operatorname{supp}(f) \text { is separable }\right\} .
$$

Recall that any subspace of a separable metrizable space is separable. Also, note that any metrizable space $X$ is completely regular, that is, if $x \in X$ and $U$ is an open neighborhood of $x$ in $X$, then there exists a continuous $f: X \rightarrow[0,1]$ such that $f(x)=1$ and $f \mid(X \backslash U) \equiv 0$.

Proposition 2.2. Let $X$ be a metrizable space. Then $C_{s}(X)$ is a closed subalgebra of $C_{b}(X)$. Furthermore, if $X$ is locally separable, then $C_{s}(X)$ is unital if and only if $X$ is separable.

Proof. To show that $C_{s}(X)$ is a subalgebra of $C_{b}(X)$, let $f, g \in C_{s}(X)$. Note that

$$
\mathrm{Coz}(f+g) \subseteq \operatorname{Coz}(f) \cup \operatorname{Coz}(g),
$$


and $\operatorname{Coz}(f) \cup \operatorname{Coz}(g)$ is separable, as it is contained in $\operatorname{supp}(f) \cup \operatorname{supp}(g)$ and the latter is so. Thus $\operatorname{Coz}(f+g)$ is separable, and then so is its closure $\operatorname{supp}(f+g)$ in $X$. That is $f+g \in C_{s}(X)$. Similarly, $f g \in C_{s}(X)$.

To show that $C_{s}(X)$ is closed in $C_{b}(X)$, let $f_{1}, f_{2}, \ldots$ be a sequence in $C_{s}(X)$ converging to some $f \in C_{b}(X)$. Note that

$$
\operatorname{Coz}(f) \subseteq \bigcup_{n=1}^{\infty} \operatorname{Coz}\left(f_{n}\right)=C
$$

and $\operatorname{Coz}\left(f_{n}\right)$ is separable for each $n$, as it is contained in $\operatorname{supp}\left(f_{n}\right)$. Thus the countable union $C$ is also separable. But then the subspace $\operatorname{Coz}(f)$ of $C$ is separable, and thus so is its closure $\operatorname{supp}(f)$ in $X$. Therefore $f \in C_{s}(X)$.

It is obvious that if $X$ is separable, then $C_{s}(X)$ is unital with the unit element 1 (the function which maps every element of $X$ to 1 ). Now suppose that $X$ is locally separable and that $C_{s}(X)$ is unital. We show that $X$ is separable. Let $u$ be the unit element of $C_{s}(X)$. Let $x \in X$. Since $X$ is locally separable, there exist a separable open neighborhood $U_{x}$ of $x$ in $X$. Let $f_{x}: X \rightarrow[0,1]$ be continuous with $f_{x}(x)=1$ and $f_{x} \mid\left(X \backslash U_{x}\right) \equiv 0$. Since $\operatorname{Coz}\left(f_{x}\right)$ is separable (as $\left.\operatorname{Coz}\left(f_{x}\right) \subseteq U_{x}\right)$, so is its closure $\operatorname{supp}\left(f_{x}\right)$ in $X$, and thus $f_{x} \in C_{s}(X)$. But then $u(x) \cdot f_{x}(x)=f_{x}(x)$ implies that $u(x)=1$. Therefore $u=\mathbf{1}$, and thus $X=\operatorname{supp}(u)$ is separable.

The following subspace of $\beta X$ will play a crucial role in our study.

Definition 2.3. For any metrizable space $X$ let

$$
\lambda X=\bigcup\left\{\operatorname{int}_{\beta X} \operatorname{cl}_{\beta X} C: C \in \operatorname{Coz}(X) \text { is separable }\right\} .
$$

Observe that $\lambda X$ coincides with $\lambda_{\mathcal{P}} X$, as defined in [6] (also, in [7] and [8]), with $\mathcal{P}$ taken to be separability (provided that $X$ is metrizable).

Note that if $X$ is a space and $D$ is a dense subspace of $X$, then $\operatorname{cl}_{X} U=\operatorname{cl}_{X}(U \cap D)$ for every open subspace $U$ of $X$; this simple observation will be used below.

Lemma 2.4. Let $X$ be a metrizable space. Then $X$ is locally separable if and only if $X \subseteq \lambda X$.

Proof. Suppose that $X$ is locally separable. Let $x \in X$, and let $U$ be a separable open neighborhood of $x$ in $X$. Let $f: X \rightarrow[0,1]$ be continuous with $f(x)=0$ and $f \mid(X \backslash U) \equiv 1$. Let $C=f^{-1}([0,1 / 2))$, then $C \in \operatorname{Coz}(X)$. (To see the latter, define

$$
g=\max \{0,1 / 2-f\}
$$

and then observe that $C=\operatorname{Coz}(g)$.) Note that $C$ is separable, as $C \subseteq U$. Since

$$
\begin{aligned}
f_{\beta}^{-1}([0,1 / 2)) & \subseteq \operatorname{cl}_{\beta X} f_{\beta}^{-1}([0,1 / 2)) \\
& =\operatorname{cl}_{\beta X}\left(X \cap f_{\beta}^{-1}([0,1 / 2))\right)=\operatorname{cl}_{\beta X} f^{-1}([0,1 / 2))=\operatorname{cl}_{\beta X} C
\end{aligned}
$$

it follows that

$$
x \in f_{\beta}^{-1}([0,1 / 2)) \subseteq \operatorname{int}_{\beta X} \operatorname{cl}_{\beta X} C \subseteq \lambda X .
$$

Now, suppose that $X \subseteq \lambda X$. Let $x \in X$. Then $x \in \lambda X$, which implies that $x \in \operatorname{int}_{\beta X} \operatorname{cl}_{\beta X} D$ for some separable $D \in \operatorname{Coz}(X)$. Let

$$
V=X \cap \operatorname{int}_{\beta X} \operatorname{cl}_{\beta X} D .
$$

Then $V$ is an open neighborhood of $x$ in $X$, and it is separable, as

$$
V \subseteq X \cap \operatorname{cl}_{\beta X} D=\operatorname{cl}_{X} D
$$


and the latter is so.

Definition 2.5. Let $X$ be a locally separable metrizable space. For any $f \in C_{b}(X)$ denote $f_{\lambda}=f_{\beta} \mid \lambda X$.

Note that by Lemma 2.4 the function $f_{\lambda}$ extends $f$.

Lemma 2.6. Let $X$ be a locally separable metrizable space. For any $f \in C_{b}(X)$ the following are equivalent:

(1) $f \in C_{s}(X)$.

(2) $f_{\lambda} \in C_{0}(\lambda X)$.

Proof. (1) implies (2). Note that $\operatorname{Coz}(f)$, being a subspace of $\operatorname{supp}(f)$, is separable. Now

and thus

$$
\operatorname{Coz}\left(f_{\beta}\right) \subseteq \operatorname{cl}_{\beta X} \operatorname{Coz}\left(f_{\beta}\right)=\operatorname{cl}_{\beta X}\left(X \cap \operatorname{Coz}\left(f_{\beta}\right)\right)=\operatorname{cl}_{\beta X} \operatorname{Coz}(f)
$$

$$
\operatorname{Coz}\left(f_{\beta}\right) \subseteq \operatorname{int}_{\beta X} \operatorname{cl}_{\beta X} \operatorname{Coz}(f) \subseteq \lambda X .
$$

For any $\epsilon>0$, the space

$$
\left|f_{\lambda}\right|^{-1}([\epsilon, \infty))=\left|f_{\beta}\right|^{-1}([\epsilon, \infty)),
$$

being closed in $\beta X$, is compact.

(2) implies (1). Let $n$ be a positive integer. Since $\left|f_{\lambda}\right|^{-1}([1 / n, \infty))$ is a compact subspace of $\lambda X$, we have

$$
\left|f_{\lambda}\right|^{-1}([1 / n, \infty)) \subseteq \operatorname{int}_{\beta X} \operatorname{cl}_{\beta X} C_{1} \cup \cdots \cup \operatorname{int}_{\beta X} \operatorname{cl}_{\beta X} C_{k}
$$

for some separable $C_{1}, \ldots, C_{k} \in \operatorname{Coz}(X)$. Intersecting both sides of (1) with $X$, it follows that $|f|^{-1}([1 / n, \infty))$, being a subspace of the separable metrizable space

$$
\mathrm{cl}_{X} C_{1} \cup \cdots \cup \mathrm{cl}_{X} C_{k},
$$

is separable. But then

$$
\operatorname{Coz}(f)=\bigcup_{n=1}^{\infty}|f|^{-1}([1 / n, \infty))
$$

is also separable, and thus so is its $\operatorname{closure} \operatorname{supp}(f)$ in $X$.

Observe that any open-closed subspace $A$ of a space $X$ is a cozero-set of $X$; indeed, $A=\operatorname{Coz}(f)$, where $f=\chi_{A}$ is the characteristic function of $A$.

Lemma 2.7. Let $X$ be a locally separable metrizable space. Let $X$ be represented as a disjoint union $X=\bigcup_{i \in I} X_{i}$, such that $X_{i}$ is a separable open-closed subspace of $X$ for each $i \in I$. Then

$$
\lambda X=\bigcup\left\{\operatorname{cl}_{\beta X}\left(\bigcup_{i \in J} X_{i}\right): J \subseteq I \text { is countable }\right\} .
$$

Proof. Denote

$$
\mu X=\bigcup\left\{\operatorname{cl}_{\beta X}\left(\bigcup_{i \in J} X_{i}\right): J \subseteq I \text { is countable }\right\} .
$$

To show that $\lambda X \subseteq \mu X$, let $C \in \operatorname{Coz}(X)$ be separable. Then $C$ is Lindelöf and therefore $C \subseteq \bigcup_{i \in J} X_{i}$ for some countable $J \subseteq I$. Thus

$$
\operatorname{cl}_{\beta X} C \subseteq \operatorname{cl}_{\beta X}\left(\bigcup_{i \in J} X_{i}\right)
$$


Next, we show that $\mu X \subseteq \lambda X$. Let $J \subseteq I$ be countable. Then $D=\bigcup_{i \in J} X_{i}$ is a cozero-set of $X$, as it is open-closed in $X$, and it is separable. Also, since $D$ is open-closed in $X$, its closure $\operatorname{cl}_{\beta X} D$ in $\beta X$ is open-closed in $\beta X$. Thus

$$
\operatorname{cl}_{\beta X} D=\operatorname{int}_{\beta X} \mathrm{cl}_{\beta X} D \subseteq \lambda X .
$$

Let $X$ be a locally compact non-compact space. It is known that $C_{0}(X)=$ $C_{00}(X)$ if and only if every $\sigma$-compact subspace of $X$ is contained in a compact subspace of $X$. (See Problem 7G.2 of [4].) In particular, $C_{0}(X)=C_{00}(X)$ implies that $X$ is countably compact, and thus non-paracompact, as every countably compact paracompact space is compact. (See Theorem 5.1.20 of [3.) Below, it will be shown that $C_{0}(\lambda X)=C_{00}(\lambda X)$ for any locally separable metrizable space $X$.

Lemma 2.8. Let $X$ be a locally separable metrizable space. Then $\lambda X$ is locally compact and $C_{0}(\lambda X)=C_{00}(\lambda X)$. In particular, $\lambda X$ is countably compact.

Proof. Note that $\lambda X$, being open in $\beta X$, is locally compact. To prove the lemma, it suffices to show that every $\sigma$-compact subspace of $\lambda X$ is contained in a compact subspace of $\lambda X$. Let $T$ be a $\sigma$-compact subspace of $\lambda X$. Then

$$
T=\bigcup_{n=1}^{\infty} T_{n}
$$

where $T_{n}$ is compact for each positive integer $n$. Assume the representation of $X$ given in Part 1.2. Using Lemma 2.7, for each positive integer $n$ (by compactness of $T_{n}$ and the fact that $\operatorname{cl}_{\beta X}\left(\bigcup_{i \in J} X_{i}\right)$ is open in $\beta X$, as $\bigcup_{i \in J} X_{i}$ is open-closed in $X$ for each countable $J \subseteq I$ ) there are countable $J_{1}^{n}, \ldots, J_{k_{n}}^{n} \subseteq I$ with

$$
T_{n} \subseteq \operatorname{cl}_{\beta X}\left(\bigcup_{i \in J_{1}^{n}} X_{i}\right) \cup \cdots \cup \operatorname{cl}_{\beta X}\left(\bigcup_{i \in J_{k_{n}}^{n}} X_{i}\right) .
$$

If we now let

$$
J=\bigcup_{n=1}^{\infty}\left(J_{1}^{n} \cup \cdots \cup J_{k_{n}}^{n}\right)
$$

then $J$ is countable, and $\operatorname{cl}_{\beta X}\left(\bigcup_{i \in J} X_{i}\right)$ is a compact subspace of $\lambda X$ containing $T$.

Let $D$ be an uncountable discrete space. Let $E$ be the subspace of $\beta D \backslash D$ consisting of elements in the closure in $D$ of countable subsets of $D$. Then $E=\lambda D \backslash D$. (Observe that separable cozero-sets of $D$ are exactly countable subspace of $D$, and each subspace of $D$, being open-closed in $D$, has open closure in $\beta D$.) In [9], the author proves the existence of a continuous (2-valued) function $f: E \rightarrow[0,1]$ which is not continuously extendible over $\beta D \backslash D$. This, in particular, proves that $\lambda D$ is not normal. (To see this, suppose, to the contrary, that $\lambda D$ is normal. Note that $E$ is closed in $\lambda D$, as $D$, being locally compact, is open in $\beta D$. By the Tietze-Urysohn Extension Theorem, $f$ is extendible to a continuous bounded function over $\lambda D$, and thus over $\beta(\lambda D)=\beta D$. But this is not possible.) This fact will be used below to show that in general $\lambda X$ is non-normal for any locally separable non-separable metrizable space $X$. This, together with Lemma 2.8, provides an example of a locally compact countably compact non-normal space $Y$ with $C_{0}(Y)=C_{00}(Y)$. 
Observe that if $X$ is a space and $D \subseteq X$, then

$$
U \cap \operatorname{cl}_{X} D=\operatorname{cl}_{X}(U \cap D)
$$

for every open-closed subspace $U$ of $X$; this simple observation will be used below.

Lemma 2.9. Let $X$ be a locally separable non-separable metrizable space. Then $\lambda X$ is non-normal.

Proof. Assume the representation of $X$ given in Part 1.2. Choose some $x_{i} \in X_{i}$ for each $i \in I$. Consider the subspace

$$
D=\left\{x_{i}: i \in I\right\}
$$

of $X$. Then $D$ is a closed discrete subspace of $X$, and since $X$ is non-separable, it is uncountable. Suppose to the contrary that $\lambda X$ is normal. Using Lemma 2.7 the space

$$
\lambda X \cap \operatorname{cl}_{\beta X} D=\bigcup\left\{\operatorname{cl}_{\beta X}\left(\bigcup_{i \in J} X_{i}\right) \cap \operatorname{cl}_{\beta X} D: J \subseteq I \text { is countable }\right\},
$$

being closed in $\lambda X$, is normal. Now, let $J \subseteq I$ be countable. Since $\operatorname{cl}_{\beta X}\left(\bigcup_{i \in J} X_{i}\right)$ is open in $\beta X$ (as $\bigcup_{i \in J} X_{i}$ is open-closed in $X$ ) we have

$$
\begin{aligned}
\operatorname{cl}_{\beta X}\left(\bigcup_{i \in J} X_{i}\right) \cap \operatorname{cl}_{\beta X} D & =\operatorname{cl}_{\beta X}\left(\operatorname{cl}_{\beta X}\left(\bigcup_{i \in J} X_{i}\right) \cap D\right) \\
& =\operatorname{cl}_{\beta X}\left(\bigcup_{i \in J} X_{i} \cap D\right)=\operatorname{cl}_{\beta X}\left(\left\{x_{i}: i \in J\right\}\right) .
\end{aligned}
$$

But $\mathrm{cl}_{\beta X} D=\beta D$, as $D$ is closed in (the normal space) $X$. Therefore

$$
\operatorname{cl}_{\beta X}\left(\left\{x_{i}: i \in J\right\}\right)=\operatorname{cl}_{\beta X}\left(\left\{x_{i}: i \in J\right\}\right) \cap \operatorname{cl}_{\beta X} D=\operatorname{cl}_{\beta D}\left(\left\{x_{i}: i \in J\right\}\right) .
$$

Thus

$$
\lambda X \cap \mathrm{cl}_{\beta X} D=\lambda D,
$$

contradicting the fact that $\lambda D$ is not normal.

A version of the classical Banach-Stone Theorem states that if $X$ and $Y$ are locally compact spaces, the Banach algebras $C_{0}(X)$ and $C_{0}(Y)$ are isometrically isomorphic if and only if the spaces $X$ and $Y$ are homeomorphic (see Theorem 7.1 of [2]); this will be used in the proof of the following main theorem.

Theorem 2.10. Let $X$ be a locally separable metrizable space. Then $C_{s}(X)$ is a $B a$ nach algebra isometrically isomorphic to the Banach algebra $C_{0}(Y)$ for some unique (up to homeomorphism) locally compact space $Y$. The space $Y$ is countably compact, and if $X$ is non-separable, is non-normal. Furthermore, $C_{0}(Y)=C_{00}(Y)$.

Proof. Let $Y=\lambda X$ and define $\psi: C_{s}(X) \rightarrow C_{0}(Y)$ by $\psi(f)=f_{\lambda}$ for any $f \in$ $C_{s}(X)$. By Lemma 2.6 the function $\psi$ is well-defined. It is clear that $\psi$ is an isometric homomorphism and $\psi$ is injective. (Note that $X \subseteq Y$ by Lemma 2.4.) Let $g \in C_{0}(Y)$. Then $(g \mid X)_{\lambda}=g$ and thus $g \mid X \in C_{s}(X)$ by Lemma 2.6. Now $\psi(g \mid X)=g$. This shows that $\psi$ is surjective. Note that by Lemma 2.8 the space $Y$ is locally compact. The uniqueness of $Y$ follows, as for any locally compact space $T$ the Banach algebra $C_{0}(T)$ determines the topology of $T$. Lemmas 2.8 and 2.9 now complete the proof. 
Remark 2.11. Theorem 2.10 holds true if one replaces "locally separable" and "the Banach algebra of continuous bounded functions with separable support", respectively, by "locally Lindelöf (locally second countable, respectively)" and "the Banach algebra of continuous bounded functions with Lindelöf (second countable, respectively) support".

Remark 2.12. By a version of the Banach-Stone Theorem, if $X$ and $Y$ are locally compact spaces, the rings $C_{0}(X)$ and $C_{0}(Y)$ are isomorphic if and only if the spaces $X$ and $Y$ are homeomorphic. (See [1].) Thus, Theorem 2.10 (and its subsequent results) holds true if one replaces "Banach algebra" by "ring".

\section{The Dimension of $C_{s}(X)$}

The Tarski Theorem states that for any infinite set $I$, there is a collection $\mathscr{A}$ of cardinality $|I|^{\aleph_{0}}$ consisting of countable infinite subsets of $I$, such that the intersection of any two distinct elements of $\mathscr{A}$ is finite (see [5]); this will be used in the following.

Note that the collection of all subsets of cardinality at most $\mathfrak{m}$ in a set of cardinality $\mathfrak{n} \geq \mathfrak{m}$ has cardinality at most $\mathfrak{n}^{\mathfrak{m}}$.

Theorem 3.1. Let $X$ be a locally separable non-separable metrizable space. Then

$$
\operatorname{dim} C_{s}(X)=d(X)^{\aleph_{0}}
$$

Proof. Assume the representation of $X$ given in Part 1.2. Note that $I$ is infinite, as $X$ is non-separable, and $d(X)=|I|$.

Let $\mathscr{A}$ be a collection of cardinality $|I|^{\aleph_{0}}$ consisting of countable infinite subsets of $I$, such that the intersection of any two distinct elements of $\mathscr{A}$ is finite. Define

$$
f_{A}=\chi_{\left(\bigcup_{i \in A} X_{i}\right)}
$$

for any $A \in \mathscr{A}$. Then, no element of

$$
\mathscr{F}=\left\{f_{A}: A \in \mathscr{A}\right\}
$$

is a linear combination of other elements (since each element of $\mathscr{A}$ is infinite and each pair of distinct elements of $\mathscr{A}$ has finite intersection). Observe that $\mathscr{F}$ is of cardinality $|\mathscr{A}|$. This shows that

$$
\operatorname{dim} C_{s}(X) \geq|\mathscr{A}|=|I|^{\aleph_{0}}=d(X)^{\aleph_{0}} .
$$

To simplify the notation, denote

$$
H_{J}=\bigcup_{i \in J} X_{i}
$$

for any $J \subseteq I$. If $f \in C_{s}(X)$, then $\operatorname{supp}(f)$ (being separable) is Lindelöf, and thus $\operatorname{supp}(f) \subseteq H_{J}$, where $J \subseteq I$ is countable; therefore, it may be assumed that $f \in C_{b}\left(H_{J}\right)$. Conversely, if $J \subseteq I$ is countable, then each element of $C_{b}\left(H_{J}\right)$ can be extended trivially to an element of $C_{s}(X)$ (by defining it to be identically 0 elsewhere). Thus $C_{s}(X)$ may be viewed as the union of all $C_{b}\left(H_{J}\right)$, where $J$ runs over all countable subsets of $I$. Note that if $J \subseteq I$ is countable, then $H_{J}$ is separable; thus any element of $C_{b}\left(H_{J}\right)$ is determined by its value on a countable 
set. This implies that for each countable $J \subseteq I$, the set $C_{b}\left(H_{J}\right)$ is of cardinality at most $2^{\aleph_{0}}$. Note that there are at most $|I|^{\aleph_{0}}$ countable $J \subseteq I$. Now

$$
\begin{aligned}
\operatorname{dim} C_{s}(X) \leq\left|C_{s}(X)\right| & \leq \mid \bigcup\left\{C_{b}\left(H_{J}\right): J \subseteq I \text { is countable }\right\} \mid \\
& \leq 2^{\aleph_{0}} \cdot|I|^{\aleph_{0}}=|I|^{\aleph_{0}}=d(X)^{\aleph_{0}},
\end{aligned}
$$

which together with the first part proves the theorem.

\section{The spectrum of the $\mathrm{C}^{*}$-Algebra $C_{s}(X)$}

In this section the underlying field of scalars is $\mathbb{C}$. Let $A$ be a commutative Banach algebra. A non-zero algebra homomorphism $\phi: A \rightarrow \mathbb{C}$ is called a character of $A$; the set of all characters of $A$ is denoted by $\Phi_{A}$. If $A$ is a $\mathrm{C}^{*}$-algebra then every algebra homomorphism $\phi: A \rightarrow \mathbb{C}$ is a $*$-homomorphism, and thus $\Phi_{A}$ coincides with the spectrum of $A$. Every character on $A$ is continuous, and therefore $\Phi_{A}$ is a subset of the space $A^{*}$ of continuous linear functionals on $A$; moreover, when equipped with the relative weak ${ }^{*}$ topology, $\Phi_{A}$ turns out to be locally compact. The space $\Phi_{A}$ is compact (in the topology just defined) if and only if the algebra $A$ has an identity element. Given $a \in A$, let the function $\hat{a}: \Phi_{A} \rightarrow \mathbb{C}$ be defined by $\hat{a}(\phi)=\phi(a)$ for any $\phi \in \Phi_{A}$. The map $a \mapsto \hat{a}$ defines a norm-decreasing, unitpreserving algebra homomorphism from $A$ to $C_{0}\left(\Phi_{A}\right)$. This homomorphism is called the Gelfand representation of $A$. In general the representation is neither injective nor surjective. The commutative Gelfand-Naimark Theorem states that if $A$ is a commutative $\mathrm{C}^{*}$-algebra then the Gelfand map is an isometric $*$-isomorphism.

Theorem 4.1. Let $X$ be a locally separable non-separable metrizable space. Then the spectrum of $C_{s}(X)$ is homeomorphic to $\lambda X$, and thus is locally compact, countably compact and non-normal.

Proof. By the commutative Gelfand-Naimark Theorem the $\mathrm{C}^{*}$-algebra $C_{s}(X)$ is isometrically $*$-isomorphic to $C_{0}(S)$, where $S$ is the spectrum of $C_{s}(X)$. On the other hand, $C_{s}(X)$ is isometrically isomorphic (as a Banach algebra) to $C_{0}(\lambda X)$, by (the proof of) Theorem 2.10. This implies that $C_{0}(S)$ is isometrically isomorphic to $C_{0}(\lambda X)$, which by the Banach-Stone Theorem (and Lemmas 2.8 and 2.9) gives the result.

\section{The Banach algebra $C_{\sigma}(X)$ of Continuous bounded functions with $\sigma-$ COMPACT SUPPORT}

Recall that in any locally compact space, $\sigma$-compactness coincides with being Lindelöf (see Problem 3.8.C of [3]); thus, in any locally compact metrizable space, $\sigma$-compactness and separability coincide.

The following variation of Theorem 2.10 might be of some interest; results dual to Theorems 3.1 and 4.1 may be stated and proved analogously.

Definition 5.1. For any metrizable space $X$ let

$$
C_{\sigma}(X)=\left\{f \in C_{b}(X): \operatorname{supp}(f) \text { is } \sigma \text {-compact }\right\} \text {. }
$$

Theorem 5.2. Let $X$ be a locally compact metrizable space. Then $C_{\sigma}(X)$ is a Banach algebra isometrically isomorphic to the Banach algebra $C_{0}(Y)$ for some unique (up to homeomorphism) locally compact space $Y$. The space $Y$ is countably compact, and if $X$ is non- $\sigma$-compact, is non-normal. Furthermore, $C_{0}(Y)=C_{00}(Y)$. 
Proof. This follows from Theorem 2.10 and the fact that $C_{\sigma}(X)=C_{s}(X)$.

Acknowledgement. The author wishes to thank the anonymous referee for his/her careful reading of the manuscript and his/her useful suggestions and comments.

\section{REFERENCES}

1. A. R. Aliabad, F. Azarpanah and M. Namdari, Rings of continuous functions vanishing at infinity. Comment. Math. Univ. Carolin. 45 (2004), 519-533.

2. E. Behrends, M-structure and the Banach-Stone Theorem. Springer, Berlin, 1979.

3. R. Engelking, General Topology. Second edition. Heldermann Verlag, Berlin, 1989.

4. L. Gillman and M. Jerison, Rings of Continuous Functions. Springer-Verlag, New YorkHeidelberg, 1976.

5. R. E. Hodel, Jr., Cardinal functions I, in: K. Kunen and J. E. Vaughan (Eds.), Handbook of Set-theoretic Topology, Elsevier, Amsterdam, 1984, pp. 1-61.

6. M. R. Koushesh, Compactification-like extensions. Dissertationes Math. (Rozprawy Mat.) 476 (2011), 88 pp.

7. M. R. Koushesh, The partially ordered set of one-point extensions. Topology Appl. 158 (2011), 509-532.

8. M. R. Koushesh, A pseudocompactification. Topology Appl. 158 (2011), 2191-2197.

9. N. M. Warren, Properties of Stone-Čech compactifications of discrete spaces. Proc. Amer. Math. Soc. 33 (1972), 599-606.

Department of Mathematical Sciences, Isfahan University of Technology, Isfahan 84156-83111, IRAN

School of Mathematics, Institute for Research in Fundamental Sciences (IPM), P.O. Box: 19395-5746, TeHran, Iran

E-mail address: koushesh@cc.iut.ac.ir 